\title{
DVB-T2 Radio Frequency Signal Observation and Parameter Correlation
}

\author{
Bexhet Kamo ${ }^{1}$, Elson Agastra ${ }^{2}$, Shkelzen Cakaj ${ }^{3}$ \\ Faculty of Information Technology, Polytechnic University of Tirana \\ Sheshi Nene Tereza 1, 1004, Tirana, Albania
}

\begin{abstract}
In this paper, field test measurement are described and statistically correlated to obtain useful information about radiofrequency (RF) behavior of Digital Video Broadcasting Terrestrial, second generation (DVB-T2) channels. Monitored radiofrequency data parameters are analyzed from statistical perspective and for finding, if any, linear correlation between them. Practical series of field measurements in the surrounding of Korça city in Albania are performed for consecutive 48 hours with sample data each second. The obtained results show the main issues that need to be considered in monitoring service reception quality which is not strongly related to the received channel power level but to the Modulation Error Rate (MER) parameter.
\end{abstract}

Keywords-DVB-T2; radio coverage; statistical correlation $R F$ data; field measurements

\section{INTRODUCTION}

Spectrum monitoring and analysis of the provided services and relative radio frequency parameters are of key importance in spectrum use planning and optimization. Spectrum use occurs 24h/day every day, likewise, spectrum monitoring should also be performed on the same continuous or statistically correct basis to ensure that the spectrum is used as intended [1].

Basic idea of this research is to create a base of real RF digital signal measurements and troubleshooting on wireless communications. This is necessary as to complete the theoretical analysis of RF signal propagations on real and complex environments [2][3].

Radiofrequency received signal parameters such SNR (Signal to Noise Ratio) are extensively used for modulation classification, signal recognition and receiver decision on correction factor to apply [4][5].

The main objective of this research is to evaluate, create a test case of a real wireless communication systems and correlate different signal parameters with each other. The purpose of this correlation is to describe the presence or not of sufficient information on RF parameters (such as SNR) to be used for classification and decision making in receiver part.

To validate the proposed approach, the wireless communication system, needs to be stable and continuous in time and not influenced by user usage. In this case, using Terrestrial Digital Video Broadcasting communications, guaranties 24 hours/day continuous signal transmitting with invariant configurations during all the monitoring time.
The VHF and UHF bands are not immune to the effects of anomalous propagation. High atmospheric pressure areas over water can give rise to ducting. Areas of unusually high ionization in the ionosphere are also prone to causing anomalous propagation effects. The result is interference from distant systems, normally considered too distant to warrant great efforts in coordination. These effects are usually transitional and while statistical data on them is available, it is only by monitoring that the implications of these anomalies on wireless systems can be judged. The appropriate interference cure will be case-specific and good monitoring data will greatly aid identifying the causes of the problem.

On DVB-T2 broadcast services and coverage area planning, spectrum monitoring process can perform measurements to check requirements. However, it should be noted that it is not possible to measure the coverage in a given area directly, but it is possible to verify the results predicted by planning tools.

With digital television systems is possible to perform many different type of measurements on either the digital bitstream prior to modulation, or on the modulated signal itself [6]. For verifying predicted service area coverage for DVB-T2 services, the measuring process will monitor modulated signal parameters such as Radio Frequency channel power level; MER (Modulation Error Rate); CNR (Carrier to Noise Ratio); BER pre-LDPC (Bit Error Rate before Low Density Parity Check block). Service providers should clearly define their understanding of coverage meaning as simple as exceeding certain field strength or more complex as end user quality of service perception. In the later case, a more complex and statistical data elaborating is required as to define a better insight of the measurement process and on the quality of service provided.

The contribution of this research is related to the monitoring process of DVB-T2 received signal at a given serviced area. Statistical analysis of the monitored signal parameters and possible correlation for measured data are performed to better understand radio frequency chain behavior. In this case, a real service area (operating in Albania since 2018 [7]) has been monitored for 48 consecutive hours with measurements every 1s for a total 172800 sample data. Performed monitoring is done on UHF channel 57 with 762 $\mathrm{MHz}$ central frequency and DVB-T2 modulator parameters as described in Table I. 
This paper is organized as follows: Section 2 describes methodology and measurements equipment setup. Section three describes test case analysis, statistical information extraction and confidence level. Detailed analysis and numerical useful data extraction are described in section four. Conclusions and recommendations for future work will be emphasized in section five.

TABLE I. DVB-T2 MODULATRO PARAMETERS FOR UHF CHANNEL 57

\begin{tabular}{|l|l|}
\hline TX modulator parameters [6][7][8] & DVB-T2 \\
\hline COFDM Mode & $32 \mathrm{k}-\mathrm{ext}$ \\
\hline Guard Interval & $1 / 32$ \\
\hline Carrier Modulation & $256 \mathrm{QAM}$ \\
\hline Pilot pattern & PP4 (TR-ACE) \\
\hline FEC & $3 / 4$ \\
\hline Net bitrate & $42.385 \mathrm{Mbps}$ \\
\hline Required C/N (Rice channel estimation) & $20.7 \mathrm{~dB}$ \\
\hline
\end{tabular}

\section{OBSeRvation Method}

Based on the ITU-R standards and recommendations a broadcast radio frequency communication is successful if some minimal quality parameters are guaranteed for certain period of time and locations, defined as percentage of the probability of availability of the communication. For this purposes and based on ITU-R recommendation [8], the monitoring site is configured as shown in Fig. 1 and parameters described below.

- $\mathrm{RX}$ antenna positioned on $10 \mathrm{~m}$ agl (above ground level)

- Three closed locations are considered for each test point to identify receiver maximum channel power

- Measurement mode: channel power

- Channel bandwidth: $8 \mathrm{MHz}$

- $\mathrm{RBW}: 40 \mathrm{kHz}$

- Detector type: r.m.s.

- Sweep time: $1 \mathrm{~s}$

- Unit: dBm

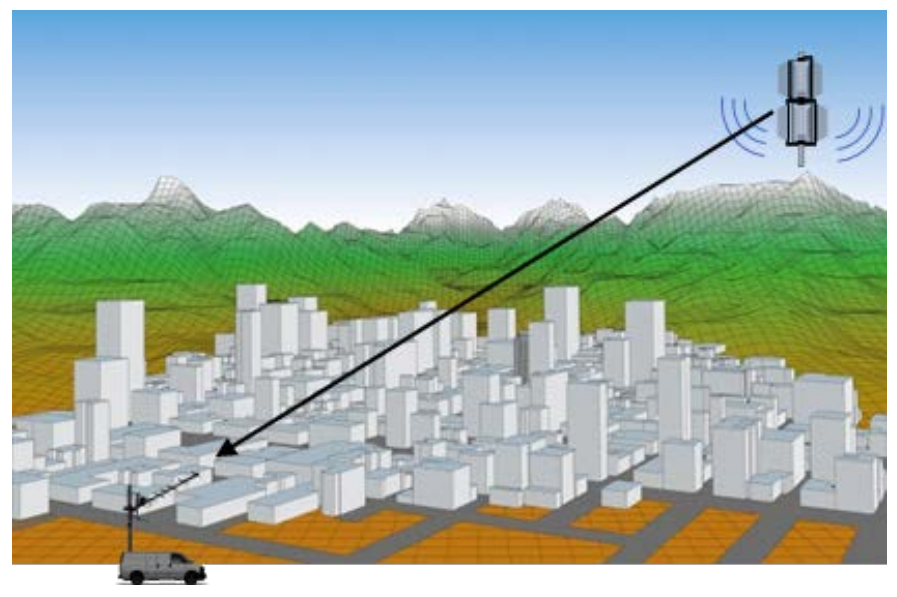

Fig. 1. Sketch of Monitoring Configuration.
The receiving antenna is a calibrated one with $11 \mathrm{dBi}$ gain in the given frequency channel. Spectrum/signal analyzer was configured to automatically save sample data every second and transfer them to the attached processing unit for further analysis.

The chosen monitoring site is line of sight with the transmitting station.

\section{TEST CASE ANALYSIS}

To validate the predicted coverage analysis of the given territory, and to have a better insight on RF system performance for DVB-T2 network, a monitoring campaign is performed in the region of Korça, Albania by the authors [7]. At each test point in the given area, a live measurement of each RF parameter is recorded automatically every $1 \mathrm{~s}$ for consecutive 48 hours in June 2019, for a total of 172800 sample data for each test site. A visual representation of the recorded data for consecutive 48 hours in one site are shown in Fig. 2.

Form the recorded data, some statistical information as mean value $(\mu)$ and relative standard deviation $(\sigma)$ for each parameter are obtained. However, as recorded data are random variables, if sample changes over time, also evaluated mean and standard deviation will change too. To better present mean $\mu$ and standard deviation $\sigma$, estimation of this parameters as $\hat{\mu}$ and $\hat{\sigma}$ as statistical variables with a confidence interval $\left(\mu_{C I}\right.$ and $\sigma_{C I}$ ) are calculated based on a percentage of confidence level. So, the expected value and relative standard deviation are evaluated, and results are shown in Table II.

The standard deviation for channel signal power $(\sim 0.7 \mathrm{~dB})$ is beyond the expected one of $3.3 \mathrm{~dB}$ as suggested in ITU-R recommendation [8] [9] for fixed reception.

Due to the nature of the observed parameters and in the scale they are presented (logarithmic), the obtained confidence level can be neglected and is comparable with instrument measuring tolerance.
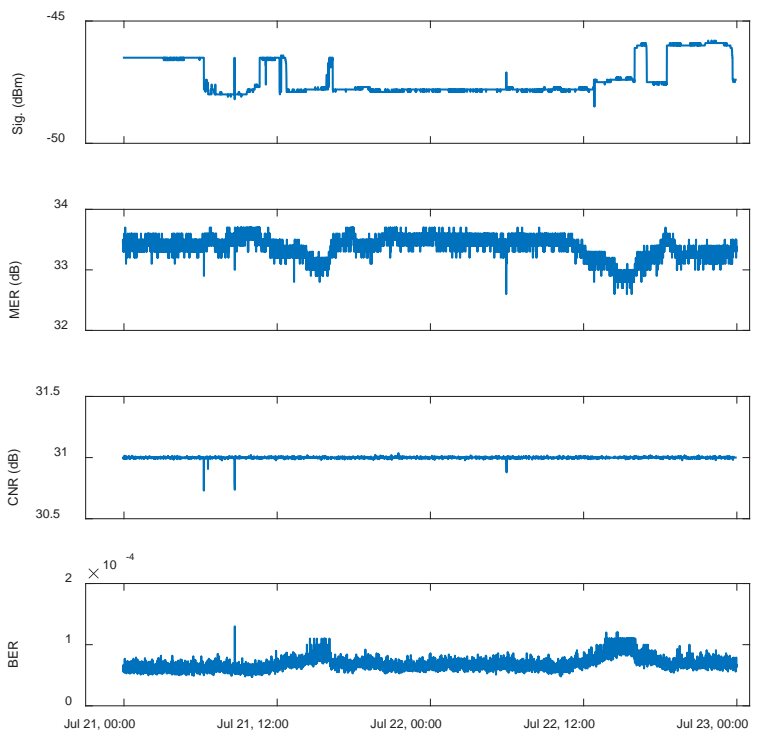

Fig. 2. Time Variation of RF Recorded Data. 
TABLE II. EXPECTED VALUE AND CONFIDENCE INTERVAL (CI) WITH 90\% CONFIDENCE LEVEL

\begin{tabular}{|l|l|l|l|l|}
\hline & $\hat{\mu}$ & $\square_{\mathrm{i}}$ & $\hat{\sigma}$ & $\square_{\mathrm{i}}$ \\
\hline Sig. $(\mathrm{dBm})$ & -47.3173 & \pm 0.0029 & 0.7134 & \pm 0.0021 \\
\hline MER $(\mathrm{dB})$ & 33.3659 & \pm 0.0008 & 0.2040 & \pm 0.0006 \\
\hline SNR $(\mathrm{dB})$ & 30.9997 & \pm 0.0002 & 0.0538 & \pm 0.0002 \\
\hline BER $\left(\times 10^{-4}\right)$ & 0.7253 & \pm 0.0021 & 0.5183 & \pm 0.0015 \\
\hline
\end{tabular}

Observed data can be presented as Normalized Probability Distribution Function (PDF) and relative Cumulative Distribution Function (CDF) as shown in Fig. 3. This information is useful to refer observations to the ITU-R coverage parameters and compare to predicted values later.

As the interests is for RF channel power level as minimum received value for which the received signal level is guaranteed on certain percentage of time (ex. $90 \%$ of time), the definition of cumulative distribution function we are interested on is the opposite of standard CDF which sees the maximum value of the statistical data. In this case, is convenient to define the cumulative probability distribution function (CPDF) as in equation (1) for RF channel power level, MER and CNR and as CDF for BER:

$$
C P D F=(1-C D F) \times 100 \%
$$

The above definition is congruent with the usage of the statistical information describing the physical phenomena. In this case, the worst case is presented for RF channel power as a minimum value, the same is true for MER and CNR and the opposite is true for BER where the worst case is presented with the maximum value.
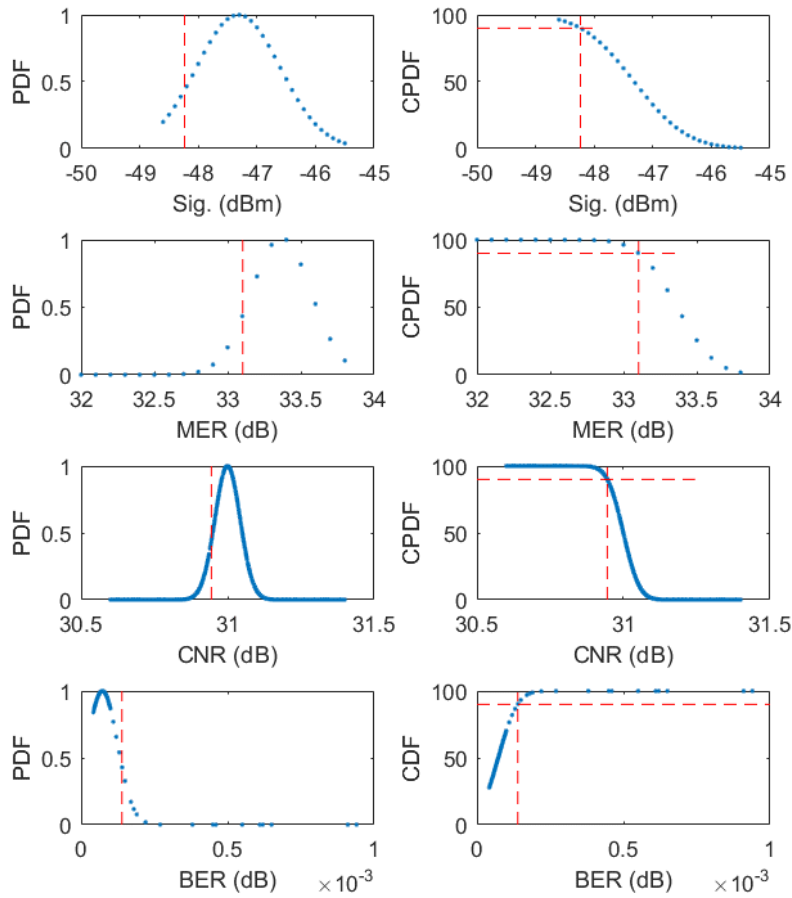

Fig. 3. Normalized Probability Density Function (PDF) and Cumulative Probability Distribution Function (CPDF) for Recorded Data. Dashed Line Highlights 90\% Probability Limit as Example.
An alternative view of the same interpretation can be evaluated from Fig. 3 where graphics regarding Signal level; MER and CNR will be read from right to left and the BER from left to right.

In this case is useful to define the coverage as percentage of time (mostly as $50 \%$ or $90 \%$ of time). So, from the observed data is possible to evaluate RF parameters with the required confidence level as highlighted in Fig. 3. In this case, if the worst-case scenario is requested for $90 \%$ of time availability, graphics presented in Fig. 3 are useful to construct data on Table III.

From Table III, $90 \%$ of samples (90\% of time) have RF channel power level greater than $-48.23 \mathrm{dBm}$; MER greater than 33.10dB; SNR greater than 30.93dB and BER lower than $1.39 \times 10^{-4}$. The same conclusion can be obtained from mean and standard deviation relative to each parameter, and statistically define the required $90 \%$ confidence level. The later can be useful to analytically compute confidence level with less sample data.

TABLE III. MEASURED RF PARAMETERS WITH 90\% TIME PROBABILITY

\begin{tabular}{|l|l|l|}
\hline & Relationship & Limit for 90\% of time \\
\hline Sig. $(\mathrm{dBm})$ & Grater than & -48.23 \\
\hline MER $(\mathrm{dB})$ & Grater than & 33.10 \\
\hline SNR $(\mathrm{dB})$ & Grater than & 30.93 \\
\hline BER $\left(\times 10^{-4}\right)$ & Smaller than & 1.39 \\
\hline
\end{tabular}

\section{RF DATA CORRELATION}

Monitored data analyzed in the previous paragraphs just presents the measure findings but no relationships are still computed between RF parameters.

In this case, statistical parameter evaluations are of critical importance, not only for the design process phase, but also on the live measurements to confirm or correct the predicted behavior on the network design phase [10][11][12][13].

All RF parameters are essential for quality DVB-T2 signal reception and decoding. In this section some statistical manipulation procedures are defined as to better understand the relationship of monitored parameters. As will be discussed later on, an increment of received channel power, not necessary will result in better CNR or MER and less BER.

For this purpose the Pearson correlation coefficient is used as a measure of correlation for two of any couple of random RF parameters. The definition we will use for Pearson correlation coefficient as presented in [14].

For the intent of this material, let $A$ and $B$ be two of any RF parameters, each with $N$ scalar observations, then the Pearson correlation coefficient $\rho(A, B)$ is defined as in equation (2) where $\mu_{A}$ and $\sigma_{A}$ are the mean and standard deviation of $A$, respectively, and $\mu_{B}$ and $\sigma_{B}$ are the mean and standard deviation of $B$.

$$
\rho(A, B)=\frac{1}{N-1} \sum_{i=1}^{N}\left(\overline{\frac{A_{i}-\mu_{A}}{\sigma_{A}}}\right)\left(\frac{B_{i}-\mu_{B}}{\sigma_{B}}\right)
$$


From this definition, a correlation coefficient matrix of two random variables which is the matrix of correlation coefficients for each pairwise variable combination is defined as in equation (3).

$R=\left(\begin{array}{ll}\rho(A, A) & \rho(A, B) \\ \rho(B, A) & \rho(B, B)\end{array}\right)$

Since any of variables are always directly correlated to themselves, the diagonal entries are just 1.

Computing Pearson correlation matrix coefficients as in equation (3) for any of pairwise of monitored RF parameters, results in data presented in Table IV.

Analyzing Table IV, data entry a very weak correlation of channel power level and $\operatorname{BER}(\rho=-0.0125$, close to 0$)$ which means that these variables are uncorrelated, but this does not mean that are independent [14].

To have a better insight of statistical behavior for RF parameters, a correlation behavior is computed and presented in graphic way for each pairwise of RF parameters and shown in Fig. 4. The later permits for visual inspection and relationship and correlation of RF parameters.

From telecommunication theory, is expected to have a strong correlation of signal channel power and carrier to noise ratio, but as can be seen from Fig. 4 it is not met in this case. Also, the same data is reported as Pearson correlation coefficient in Table IV, where the correlation coefficient is too weak (0.0041, close to 0 ) which means that these two variables are uncorrelated, but this does not mean that are independent.

From visual analysis, some linear correlation of BER with MER are observed, in this case negative correlation as reported in Table IV (-0.6132). The negative value indicates that one variable increases its value, the other decreases its relative value and vice-versa. Also, this is what is expected, as better MER (higher values) will result in less de-modulations errors and in less BER. This is not true for CNR and BER where a correlation coefficient of -0.0077 is observed. The negative sign of Pearson correlation coefficient is coherent with the expectations, but not its absolute value.

For the DVB-T2 modulation parameters used on this implementation, and in the analyzed location, is always possible to decode the DVB-T2 transport stream without any error for the quality of received RF parameters.

TABLE IV. CORRELATION COEFFICIENTS FOR PAIRS OF RF DATA

\begin{tabular}{|l|l|l|l|l|}
\hline & Sig. & MER & CNR & BER \\
\hline Sig. & 1 & -0.2567 & 0.0041 & -0.0125 \\
\hline MER & -0.2567 & 1 & 0.0033 & -0.6132 \\
\hline CNR & 0.0041 & 0.0033 & 1 & -0.0077 \\
\hline BER & -0.0125 & -0.6132 & -0.0077 & 1 \\
\hline
\end{tabular}

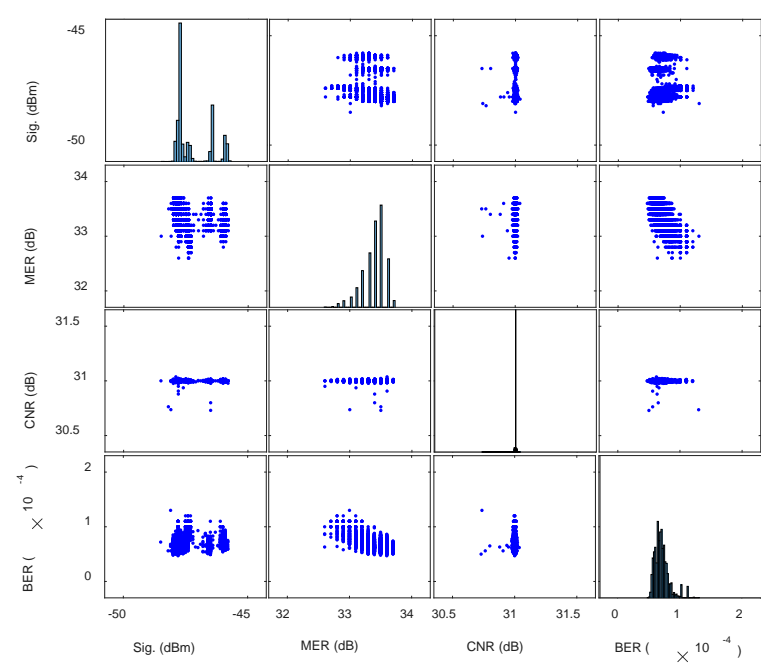

Fig. 4. Visual Correlation of RF Measured Data.

\section{CONCLUSIONS AND FUTURE WORK}

In this paper is presented radio frequency parameter monitoring for DVB-T2 network. The measuring parameters and relative standard deviations are coherent with that suggested by ITU-R for fixed reception especially the standard deviations of monitored data. Weak correlation of RF parameters are observed which indicates that during monitoring process all the RF parameters need to be temporary recorded and analyzed. Using only few of these parameters for signal/modulation classification are not sufficient. Also, received channel power is not sufficient to characterize the transmitting channel or reception signal quality. For the later, is better evaluating MER as has a higher correlation coefficient with BER and consecutively with reception signal quality from end user perspective.

As future work will be integrating and correlating monitored data for more channel frequencies at the same location and time as to better understand propagation channel behavior and to use this information for channel modeling corrections.

\section{REFERENCES}

[1] ITU-R, "Handbook on National Spectrum Management," 2015 Edition.

[2] Q. Chen, A.L. Gerig, U. Techavipoo, J. A. Zagzebski, T. Varghese, "Correlation of RF signals during angular compounding," IEEE Transactions on Ultrasonics, Ferroelectrics and Frequency Control, 2005, 52(6), 961-970.

[3] A. P. G. Hoeks, T. G. J. Arts, P. J. Brands, R. S. Reneman, "Comparison of the performance of the RF cross correlation and doppler autocorrelation technique to estimate the mean velocity of simulated ultrasound signals," Ultrasound in Medicine \& Biology, 1993, 19(9), 727-740.

[4] A. Hazza, M. Shoaib, S.A. Alshebeili, A. Fahad, "An overview of feature-based methods for digital modulation classification," 1st International Conference on Communications, Signal Processing, and Their Applications (ICCSPA) 2013.

[5] S. Hassanpour, A. M. Pezeshk and F. Behnia, "A robust algorithm based on wavelet transform for recognition of binary digital modulations," 2015 38th International Conference on Telecommunications and Signal Processing (TSP), Prague, 2015, pp. 508-512.

[6] ETSI Technical Report (ETR 290), "Digital Video Broadcasting (DVB); Measurement Guidelines for DVB Systems,” May 1997. 
[7] B. Kamo, E. Agastra and S. Cakaj, "DVB-T2 Coverage Area in Albanian Allotments using existing Analog TV Transmitting Antennas," 2018 26th IEEE International Conference on Software, Telecommunications and Computer Networks (IEEE-SoftCOM), Split, 2018, pp. 1-5.

[8] ITU-R Recommendation SM.1875-2, "DVB-T coverage measurements and verification of planning criterua," August 2014.

[9] I. Eizmendi, G. Prieto, G. Berjon-Eriz, I. Landa and M. Velez, "Empirical DVB-T2 Thresholds for Fixed Reception," in IEEE Transactions on Broadcasting, vol. 59, no. 2, pp. 306-316, June 2013.

[10] K. Ruščić and A. Skenderović, "Measurements and propagation model tuning in DVB-T2 network," Proceedings ELMAR-2014, Zadar, 2014, pp. 1-4.
[11] A. Martian, M. Dambeanu, C. Oprea, C. Vladeanu and I. Marghescu, "DVB-T2 radio coverage analysis in Romania," 2017 25th Telecommunication Forum (TELFOR), Belgrade, 2017, pp. 1-4.

[12] M. Slimani et al., "Results of the DVB-T2 Field Trial in Germany," in IEEE Transactions on Broadcasting, vol. 61, no. 2, pp. 177-194, June 2015.

[13] B. Ruckveratham and S. Promwong, "Performance evaluation of DVBT2 propagation for fixed reception," 2016 13th International Conference on Electrical Engineering/Electronics, Computer, Telecommunications and Information Technology (ECTI-CON), Chiang Mai, 2016, pp. 1-5.

[14] Joaquim P. Marques de Sá, "Applied Statistics Using SPSS, STATISTICA, MATLAB and R,” $2^{\text {nd }}$ edition, Springer-Verlag, 2007 